\title{
Performance and Computational Complexity Comparison of Block-Based Fingerprint Enhancement
}

\author{
Suksan Jirachaweng, Teesid Leelasawassuk, and Vutipong Areekul \\ Kasetsart Signal \& Image Processing Laboratory (KSIP Lab), \\ Department of Electrical Engineering, Kasetsart University, Bangkok, 10900, Thailand \\ g4885038@ku.ac.th, g4765176@ku.ac.th, vutipong.a@ku.ac.th
}

\begin{abstract}
Performance and computational complexity comparisons of various block-based fingerprint enhancement schemes are tested and reported in this literature. Enhancement performance is evaluated by comparing equal error rates, which obtained by a proposed fingerprint matching algorithm using local and global features. Various enhancement methods are tested; i.e. three types of spatial Gabor filtering, short-time Fourier transform filtering, and discrete cosine transform filtering. These enhancement schemes also tested with various databases such as FVC2000, FVC2002, and FVC2004. Finally, computational complexity of enhancement implementation is analyzed and concluded.
\end{abstract}

Keywords: Fingerprint Enhancement, Fingerprint Enhancement Performance Comparison, Spatial-Domain/Frequency-Domain Fingerprint Enhancement, Fingerprint Enhancement Computational Complexity.

\section{Introduction}

Inevitably, many fingerprint identification applications are playing an important role in our everyday life from personal access control to global border control. To pursue this goal, automatic fingerprint identification system (AFIS) must be proved to be highly reliable and flexible. Since most AFISs are based on minutiae matching, these systems rely on good quality of input fingerprint images for minutiae and ridge extraction. Unfortunately, poor quality of fingerprint is now a major problem for most AFISs. In order to reduce this problem, fingerprint enhancement is required to improve quality of fingerprint in order to precisely extract minutiae [1].

The need for superb enhancement is inevitable for poor quality fingerprints, where recoverable regions contain necessary features for matching. However, processing limitation of devices, such as embedded system or smart card with limited memory size, confines computational complexity of enhancement algorithm. Therefore, tradeoff between enhancement performance and computational complexity is also one of interesting problems for practical implementation of fingerprint recognition.

There are many fingerprint enhancement algorithms in literature. Scope of this paper is focused on widely used block-based contextual filtering, which can be roughly classified into two major types; i.e. spatial-domain and frequency-domain. In spatialdomain approaches, Gabor filtering plays an important role [2]. This method employs a set of pre-computed Gabor filters that selected by local ridge orientation and local 
ridge frequency in order to perform 2-D convolution with an entire image. In addition, computational complexity of this Gabor filtering can be reduced by applying separable Gabor filters as reported in $[3,4]$. In frequency-domain approaches, Gabor filter can be implemented in Short Time Fourier Transform (STFT) domain, resulting in better enhancement around high curvature zone [5]. Enhancement can be applied in Discrete Cosine Transform (DCT) domain [6]. The STFT and DCT approaches take advantage of frequency domain, which could design arbitrary 2-D filter shape, corresponding to improve quality of fingerprint in high curvature zones.

Goal of this paper is to compare block-based fingerprint enhancement of various schemes, of various domains, and among various fingerprint databases, in term of recognition performance and computational complexity comparison.

\section{Fingerprint Enhancement Problems}

To compare enhanced quality of fingerprint subjectively, 4 important properties of block-based fingerprint enhancement should be investigated; i.e. high curvature ridge preservation, intrinsic estimation accuracy, poor quality ridge recoverability, and spurious minutia avoidance. These four cases are discussed as follows.

Firstly, high curvature area always suffers from partitioning. In spatial domain, Gabor filter enhancement assumes that each block contains only unidirectional ridges, resulting in blocking artifacts at boundary around high curvature area. On the other hand, frequency domain approaches (STFT \& DCT) take advantage of 2-dimensional filter design with arbitrary shaping, as shown in Fig 1/(1)/(b, c, d).

Secondly, ridge intrinsic plays an important role in fingerprint recognition such as filtering, global orientation modeling, fingerprint classifying, and fingerprint matching. Ridge intrinsic estimation can be done in both spatial and frequency domains. For spatial-domain approach, ridge orientation is estimated based on the relative between horizontal gradient and vertical gradient, and then ridge frequency is estimated based on gray ridge projection along ridge's direction. Hence, accuracy of the ridge frequency will depend on accuracy of the ridge orientation estimation that computed previously. For frequency-domain approach, ridge orientation and ridge frequency parameters are simultaneously estimated from the highest peak coefficients of spectrum. However, with low quality fingerprint, the highest peak could not pinpoint. For example, Fig $1 /(2 \& 3) /(b, c, d)$ show error results from ridge orientation estimation and ridge frequency estimation, respectively.

Thirdly, poor quality ridge recoverability is one of significant indicator of fingerprint enhancement performance. Consider original oily fingerprint, shown in Fig 1/(4)/(a), enhanced with 3 different methods, resulting in Fig 1/(4)/(b, c, d). The Fourier approach performed best enhancement in oily ridge region because of two reasons; i.e. 1) Fourier domain approach preserves energy normalization, resulting in adaptive ridge's contrast without interfering DC coefficient. 2) 2D Fourier bases are based on parallel lines pattern of different frequencies in vertical and horizontal orientation. This domain is very suitable for parallel ridges shaping with Gabor filter. Unlike Fourier, 2-D DCT bases are similar to chess board patterns, and it is not suitable for ridge enhancement in general. 

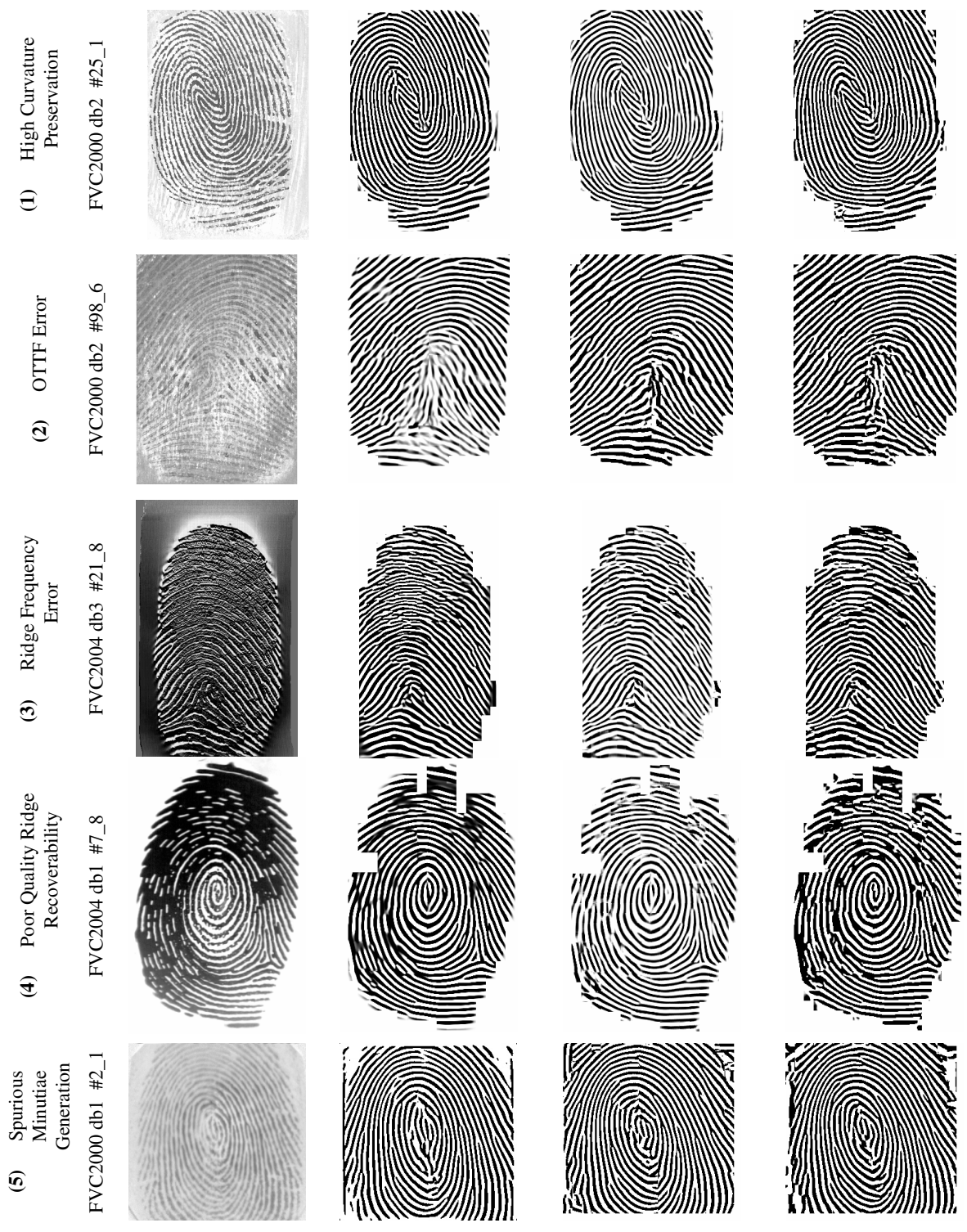

(a) Original Fingerprint

(b) Separable Gabor

(c) STFT

(d) DCT

Fig. 1. Various problems of fingerprint enhancement with different domain approach (a) Original fingerprint from FVC2000 and FVC2004 (b) Enhanced results from separable Gabor in spatial domain[4], (c) Enhanced results from STFT approach modified from [5] in frequency domain, (d) Enhanced results from DCT approach method [6] in frequency domain

The last problem relates to capability of spurious minutia avoidance. In case of segmentation error or noisy and dirty fingerprint, frequency domain approaches, which has advantage of poor ridge recoverability, generates fake ridges and spurious 
minutia in this area, as shown in Fig $1 /(5) /(b, c, d)$. This is clearly a drawback of frequency domain approaches. In conclusion, subjective performance comparison of three fingerprint enhancement approaches due to four major problems is summarized in the Table 1.

Table 1. Summary of Subjective Performance Comparison among three Fingerprint Enhancement Algorithms due to four Important Properties of Fingerprint Enhancement

\begin{tabular}{lccc}
\hline Capabilities & $\begin{array}{c}\text { Gabor Filtering } \\
\text { (Spatial) }\end{array}$ & $\begin{array}{c}\text { STFT } \\
\text { (Frequency) }\end{array}$ & $\begin{array}{c}\text { DCT } \\
\text { (Frequency) }\end{array}$ \\
\hline High Curvature Ridge Preservation & Poor & Good & Good \\
Accuracy of Intrinsic Parameter Estimation & Fair & Good & Good \\
Poor quality Ridge Recoverability & Fair & Good & Poor \\
Spurious Minutia Avoidance & Good & Poor & Poor \\
\hline
\end{tabular}

\section{Effect of Enhancement on Fingerprint Recognition}

The simply way to test enhancement performance is by testing their results with the same fingerprint matching algorithm. A fingerprint matching algorithm was developed based on three major features; focal point as singular point [7], orientation fields, and minutiae. This matching algorithm has been locally reported in $[8,9]$ and its concept is reviewed for clear explanation as the following.

A focal point, similar to a core point, can be used as a reference point for fingerprint registration and recognition. The concept of this matching algorithm is to reorganize minutiae features around the focal point as 1-Dimensional feature sequence. Minutiae features can be categorized into single minutiae feature and couple minutiae feature. The single minutia feature consists of $\left(x_{i}, y_{i}\right)$ coordinate of the $i^{\text {th }}$ minutia in fingerprint image, $\theta_{i}$ minutia-tail direction, and $t_{i}$ minutia type (end or bifurcation), as shown in Fig 2 (a). The couple minutiae feature consists of $d_{i j}$ distance between minutiae $i^{\text {th }}$ and $j^{\text {th }}, \alpha_{I}$ and $\alpha_{j}$ relative angles between a minutiae pair, and $r$ ridge count. The double minutiae features are demonstrated in Fig 2 (b).

To arrange minutiae into a 1-D feature sequence, in case of focal point existence, minutiae features are arranged around the focal point. The order of minutiae depends on their position and orientation, respected to the focal point. By using the vertical axis as a zero degree reference as shown in Fig. 2 (c), the $1^{\text {st }}$ single minutia is a head of sequence, following by the $2^{\text {nd }}$ minutia with counterclockwise search, resulting in a looped minutiae sequence in Fig. 2(d). In case of missing focal point, single minutia can be ordered by its tail direction. By using the vertical axis as a zero degree reference, each minutia tail direction can be arranged in order from zero to 360 degree. This single minutia sequence should be unique for each fingerprint if tail direction is perfectly detected. Fig. 2(e) shows actual links of a looped minutiae vector sequence without the focal point. 


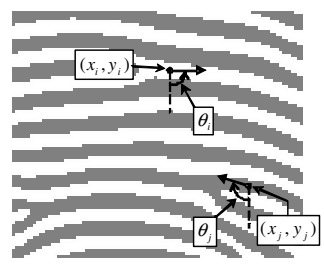

(a)

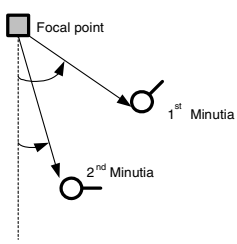

(c)

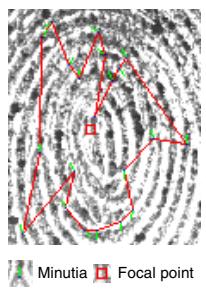

(d)

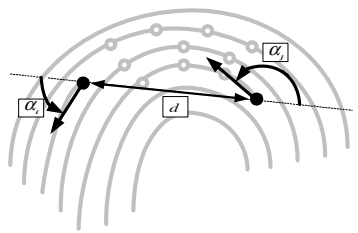

(b)

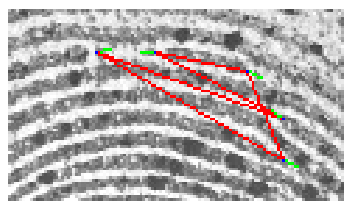

(e)

Fig. 2. Minutiae features and ordering: (a) Single minutia features. (b) Double minutiae features (c) Two minutiae ordering. (d) Actual looped minutiae sequence with a focal point. (e) Actual looped minutiae sequence without focal point.

After finishing looped minutiae arrangement, the next step is to generate the double minutiae features from the looped sequence of single minutia. By coupling two nearest neighbor minutiae in the looped sequence, a pair of minutiae can be formed and the double minutiae feature can be generated as shown in Fig 2(b). For example, the first minutia is paired with the second minutia in the looped sequence. The second minutia is coupled with the third, and so on.

A matching process composes of two stages; initial sequence alignment and minutiae sequence matching. Detail of this matching algorithm is fully explained in [9]. Matching concept is briefly explained as follows.

\section{Step 1) Initial Sequence Alignment}

- Select each minutia from both sequences, use a minutia point as a reference point, and correlate all orientation fields around minutia point and select a few minutiae couple from both sequences with highly correlative scores.

a From these few selected minutiae, find a few nearest neighbor minutiae and test with similarity score of single minutiae features and double minutiae features. In case of focal point existence, few double minutiae feature vectors can be traced and checked with a focal point. In case of no focal point, all minutiae vectors are tested with similarity score.

- Select the couple minutiae with the highest similarity score to be an initial head vector.

\section{Step 2) Minutiae Vector Sequence Matching}

- In case of no focal point input comparison, template sequence must pregenerate minutiae loop sequence without a focal point in order to prepare comparison to non-focal point input sequence. 
Use the initial head vector from step 1 for synchronizing both sequences. Then apply dynamic programming through both sequences and count matching minutiae vectors. Convert it to matching score.

The experimental results have been evaluated on public fingerprint database FVC2000, FVC2002, and FVC2004 [1,10] (100 fingers, 8 fingerprints each) in term of equal error rate. The fingerprint image is partitioned into blocks of $16 \times 16$ pixels, and a basic segmentation scheme using mean and variance is employed. Five fingerprint enhancement filtering types are evaluated as follows; Traditional Gabor filtering with non-quantized orientation (TG) [2], Separable Gabor filtering with 8-quantized orientation (SG8) [3], Separable Gabor filtering with non-quantized orientation (SG) [4], Short Time Fourier Transform approach (STFT) [5], and Discrete Cosine Transform approach (DCT) [6]. In the spatial domain approaches, the discrete Gabor filters are the same $25 \times 25$ fixed-window size. For the STFT and the DCT approaches in frequency domain, fingerprint image is also partitioned into $16 \times 16$ blocks but each block is transformed with $32 \times 32$ overlapped window in order to reduce blocking artifacts. Note that the probability estimation in [5] is not included. Finally, enhancement results are tested with our minutiae matching algorithm, and equal error rate (EER) is reported in Table 2.

Table 2. Summary of verification performance comparison among five fingerprint enhancement algorithms with FVC 2000, FVC 2002, and FVC 2004 Fingerprint Databases

\begin{tabular}{ccccccccccccc}
\hline Enhanced & \multicolumn{10}{c}{ Average Equal Error Rate in Percent (\%) } \\
\cline { 2 - 13 } Algorithms & \multicolumn{1}{c}{ FVC2000 } & \multicolumn{1}{c}{ FVC2002 } & \multicolumn{7}{c}{ FVC2004 } \\
& DB1a & DB2a & DB3a & DB4a & DB1a & DB2a & DB3a & DB4a & DB1a & DB2a & DB3a & DB4a \\
\hline TG [2] & N/A & 1.63 & 6.28 & $\mathbf{4 . 3 8}$ & 5.09 & 3.89 & 9.71 & 3.85 & $\mathbf{6 . 3 0}$ & 8.14 & 6.84 & $\mathbf{6 . 4 8}$ \\
SG [3] & N/A & 2.04 & 6.40 & 4.56 & 5.89 & 3.92 & 8.57 & $\mathbf{3 . 8 3}$ & 6.53 & 7.98 & 6.95 & 6.50 \\
SG8 [4] & N/A & 2.98 & 6.93 & 6.17 & 5.43 & 5.09 & 12.1 & 5.38 & 8.36 & 9.28 & 8.63 & 6.84 \\
STFT [5] & N/A & 1.23 & 5.75 & 7.06 & 5.28 & 3.46 & 7.45 & 4.92 & 6.75 & $\mathbf{7 . 5 0}$ & $\mathbf{5 . 3 9}$ & 7.73 \\
DCT [6] & N/A & $\mathbf{1 . 1 1}$ & $\mathbf{5 . 0 6}$ & 5.98 & $\mathbf{4 . 3 8}$ & $\mathbf{3 . 1 0}$ & $\mathbf{6 . 8 4}$ & 4.08 & 6.75 & 10.3 & 6.74 & 6.82 \\
\hline
\end{tabular}

Note that N/A is not available, due to our technical execution problem with segmentation.

\section{Computational Complexity Analysis and Comparison}

In order to compare computational complexity among various enhancement algorithms (Spatial Gabor Filtering, Short Time Fourier Transform (STFT), and Discrete Cosine Transform (DCT)), computational cost is measured in term of a number of additions $\left(\mathrm{N}^{+}\right)$and multiplications $\left(\mathrm{N}^{\times}\right)$per one partitioned block, with size $W \times W$ pixels. Assume that one partitioned window has only one dominant ridge orientation. Enhancement consists of intrinsic estimation process and filtering process. However, focal point localization process is not included.

\subsection{Spatial Gabor Filtering Approach}

1-D band-pass Gabor filtering for 8 directions (SG8) [3] is analyzed. This algorithm is composed of three processes. 


\subsubsection{Ridge Orientation Estimation}

Ridge orientation estimation is obtained by summation of $V_{\mathrm{x}}$ and $V_{\mathrm{y}}$ gradients in horizontal and vertical directions respectively. These two gradients are obtained by convolution with horizontal and vertical mask filtering $(3 \times 3$ pixels). Then ridge orientation can be estimated by $\theta=\tan ^{-1}\left(V_{\mathrm{x}} / V_{\mathrm{y}}\right)$. Computational complexity is summarized in Table 3 .

Table 3. Number of the Additions and Multiplications in Gradients Operating

\begin{tabular}{cccccc}
\hline & $G_{x}$ & $G_{y}$ & $V_{x}=2 \sum \sum\left(G_{x} G_{y}\right)$ & $V_{y}=\sum \sum\left(G_{x}{ }^{2}-G_{y}{ }^{2}\right)$ & Total \\
\hline $\mathrm{N}^{+}$ & $9 W^{2}$ & $9 W^{2}$ & $W^{2}+1$ & $W^{2}$ & $20 W^{2}+1$ \\
$\mathrm{~N}^{\times}$ & $9 W^{2}$ & $9 W^{2}$ & $W^{2}$ & $2 W^{2}$ & $21 W^{2}$ \\
\hline
\end{tabular}

\subsubsection{Ridge Frequency Estimation}

Ridge frequency can be directly estimated in frequency domain. Computational complexity is reported in term of $\left[N^{+}, N^{\times}\right]$, a number of addition and multiplication respectively. Algorithm consists of three main steps: Step 1): all gray ridge pixels in the block are projected into $1 \mathrm{D}$ sequence along ridge orientation, which requires $\left[W^{2}, 0\right]$. Step 2): the projected sequence is transformed to frequency domain, which requires $\left[\mathrm{FFT}_{1 \mathrm{D}}{ }^{+}(W), \mathrm{FFT}_{1 \mathrm{D}}{ }^{\times}(W)\right]$, where $\mathrm{FFT}_{1 \mathrm{D}}{ }^{+}(n)$ and $\mathrm{FFT}_{1 \mathrm{D}}{ }^{\times}(n)$ denote the number of addition and multiplication of $\mathrm{FFT}_{1 \mathrm{D}}$ (Radix 2) as described in Table 4. Step 3): ridge frequency is estimated from a distance between origin and the highest peak spectrum, which requires $[W, 2 W]$.

\subsubsection{Filtering Process}

In order to estimate Gabor filtering computational operation, filtering process is split into two consecutive phases. For the first phase, an 1-D band-pass Gabor filter, size $L$, convolved with all pixels in extended block zone, size $W(W+L-1)$, along the direction perpendicular to ridge orientation. The second phase performs convolution with an 1D Gabor low-pass filter for all pixels in the block, total $W^{2}$ pixels, along ridge direction. Implementation detail is clearly explained in [3]. Since all Gabor filters are symmetry, filtering requires only $\lfloor L / 2\rfloor$ additions and $\lfloor L / 2\rfloor+1$ multiplications. Total numbers of additions and multiplications are equal to $\left(W(W+L-1)+W^{2}\right) \times\lfloor L / 2\rfloor$ and $\left(W(W+L-1)+W^{2}\right) \times(\lfloor L / 2\rfloor+1)$, respectively.

\subsection{Frequency Approach}

In general, fingerprint enhancement in frequency domain utilizes overlapping window in order to preserve ridge continuity, and to eliminate blocking artifacts. Computation of each stage is explained as follows,

\subsubsection{Overlapping Decomposition/Reconstruction}

In general, spectrum of fingerprint's ridges locates in a specific frequency band. Other frequency bands are considered as noise, which could be eliminated in filtering process. In practice, only specific ridge frequency band is transformed and other frequency bands are ignored in order to reduce unnecessary computation, and to preserve memory.

To further reduce computational complexity, redundancy in overlapping window of $\mathrm{FFT}^{\mathrm{H}}{ }_{2 \mathrm{D}}$ and $\mathrm{DCT}^{\mathrm{H}}{ }_{2 \mathrm{D}}$ can be removed. Basically, two dimensional $\mathrm{FFT}_{2 \mathrm{D}}$ and $\mathrm{DCT}_{2 \mathrm{D}}$ can be implemented by using separable Fast Fourier transform $\left(\mathrm{FFT}_{1 \mathrm{D}}\right)$ and separable Fast Discrete Cosine transform $\left(\mathrm{DCT}_{1 \mathrm{D}}\right)$. The separable transform applies 
1-D transform along the vertical as shown in Fig 3(a) (left), and then another same 1-D transform along the horizontal as shown in Fig 3(a) (right). Assume that overlapping window size is $2 W \times 2 W$ pixels, only a quarter at the center of total FFT coefficient's area, $(W \times W)$, is related to ridge intrinsic estimation as shown in Fig 3(b), and only $W \times W$ pixels on the top-left DCT coefficients are significant as shown in Fig 3(c).

Therefore, redundancy can be further reduced by performing convolution along gray zone of all blocks in a row as shown in Fig. 3(d). For example, consider the block $B_{2}$, a half number of transformed columns can be shared with the previous block $\mathrm{B}_{1}$ and the next block $\mathrm{B}_{3}$ as shown in Fig. 3(e) for $\mathrm{STFT}_{2 \mathrm{D}}^{\mathrm{H}}$ and Fig. 3(f) for $\mathrm{DCT}_{2 \mathrm{D}}^{\mathrm{H}}$. This technique reduces computation by 4 times, $\left\{\mathrm{FFT}_{2 \mathrm{D}}(2 n \times 2 n)=\right.$ $\left.(4) \mathrm{FFT}^{\mathrm{H}}{ }_{2 \mathrm{D}}(2 n \times 2 n)\right\}$, as shown in Table 4 .

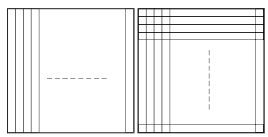

(a)

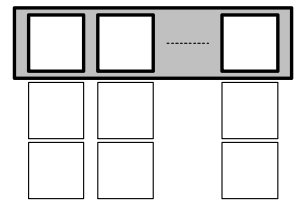

(d)

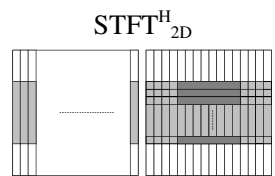

(b)

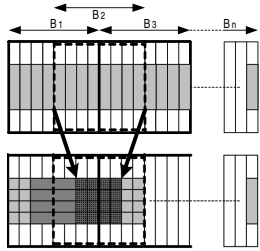

(e)

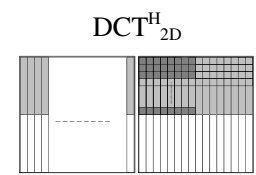

(c)

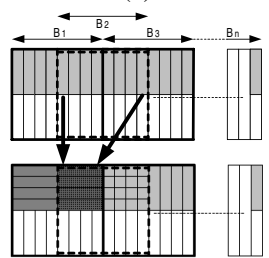

(f)

Fig. 3. Separable transforms (a) Traditional separable transform by vertical and horizontal 1-D transform, (b) Separable FFT, (c) Separable DCT transform, (d) Block transform in row, (e) Implemented $\mathrm{STFT}^{\mathrm{H}}{ }_{2 \mathrm{D}}$ for fingerprint enhancement, and (f) Implemented $\mathrm{DCT}^{\mathrm{H}}{ }_{2 \mathrm{D}}$ for fingerprint enhancement

Table 4. Number of addition and multiplication requirement for different transform types

\begin{tabular}{|c|c|c|}
\hline FFT, DCT (Radix 2) & + (Addition) & $\times$ (Multiplication) \\
\hline $\mathrm{FFT}_{1 \mathrm{D}}(n)$ & $(6 n)\left(\log _{2} n\right)$ & $(4 n)\left(\log _{2} n\right)$ \\
\hline $\mathrm{FFT}_{2 \mathrm{D}}(n \times n)$ & $(2 n) \mathrm{FFT}_{1 \mathrm{D}}^{+}(n)$ & $(2 n) \operatorname{FFT}_{1 \mathrm{D}}^{\times}(n)$ \\
\hline $\mathrm{DCT}_{2 \mathrm{D}}(n \times n)$ & $(2 n) \mathrm{DCT}^{+}{ }_{1 \mathrm{D}}(n)$ & $(2 n) \mathrm{DCT}_{1 \mathrm{D}}^{\times}(n)$ \\
\hline Overlap & + & $\times$ \\
\hline $\mathrm{DCT}_{2 \mathrm{D}}(2 n \times 2 n)$ & $(4 n) \mathrm{DCT}_{1 \mathrm{D}}^{+}(2 n)$ & $(4 n) \mathrm{DCT}^{\times}{ }_{1 \mathrm{D}}(2 n)$ \\
\hline $\operatorname{DCT}^{\mathrm{H}}{ }_{2 \mathrm{D}}(2 n \times 2 n)$ & $(n) \mathrm{DCT}_{1 \mathrm{D}}^{+}(2 n)$ & $(n) \mathrm{DCT}_{1 \mathrm{D}}^{\times}(2 n)$ \\
\hline
\end{tabular}

\subsubsection{Intrinsic Parameter Estimation}

Since ridge frequency and ridge orientation parameters can be easily estimated from a distance and an angle between zero frequency coefficient and highest peak frequency position. For FFT approach, each spectrum magnitude calculation requires one addition and two multiplications because of complex number coefficient. Since Fourier coefficients are symmetry. Therefore, a total required number of addition and multiplication 
for spectrum magnitude calculation are only $\left(W^{2}\right) / 2$ and $\left(2 W^{2}\right) / 2$, respectively. For DCT approach, coefficient is real and no computation needs because spectrum can be referred directly to its absolute value. However, DCT approach needs an additional phase analysis to classify ridge orientation [6]. This step requires two 2 -D filters with size of $5 \times 3$ pixels for calculating average directional strength. Then, it requires totally $2(5 \times 3)$ additions and 2 multiplications for normalization.

\subsubsection{Filtering}

For STFT approach, it requires two steps; frequency filtering and angular filtering. A band-pass filter in circular shape, with $r$-pixel radius, is applied directly by point to point multiplication in frequency domain. Outside filter region is forced to be zero without any operation. Therefore, the total number of multiplications is $2 \times 2 \times\left\lfloor\pi r^{2} \downarrow / 2\right.$. The first term represents a number of filtering time (frequency filtering and directional filtering). The second term represents a number of complex dimension (Real and Imaginary). The $\left\lfloor\pi r^{2}\right\rfloor$ is an approximate size of band region. The last term represents computational reduction from a symmetry property.

For DCT approach, filtering process consists of two arithmetic operations; multiplication and convolution. The multiplication needs for frequency-domain filtering and its computational complexity is similar to the FFT approach. The convolution composes of $(r+l-1) \times r \times\lfloor l / 2\rfloor$ additions and $(r+l-1) \times r \times(\lfloor l / 2\rfloor+1)$ multiplications, where $l$ represents a diagonal filter length. The first term $(r+l-1)$ and second term $r$ represent a number of filtering convolution in a major direction and a minor direction of phase flow (45 or 135 degree) in DCT domain, respectively. The last term with $\lfloor l / 2\rfloor$ and $(\lfloor/ / 2\rfloor+1)$ represents a number of additions and multiplications in each step of translation in convolution [6].

Finally, the total number of additions and multiplications of each stage are summarized in the Table 5 . Note that (.)* shows computational complexity results under our experimental condition with $W=16, L=25, r=10$ and $l=5$.

Table 5. Number of addition and multiplication requirement for various fingerprint enhancement processes

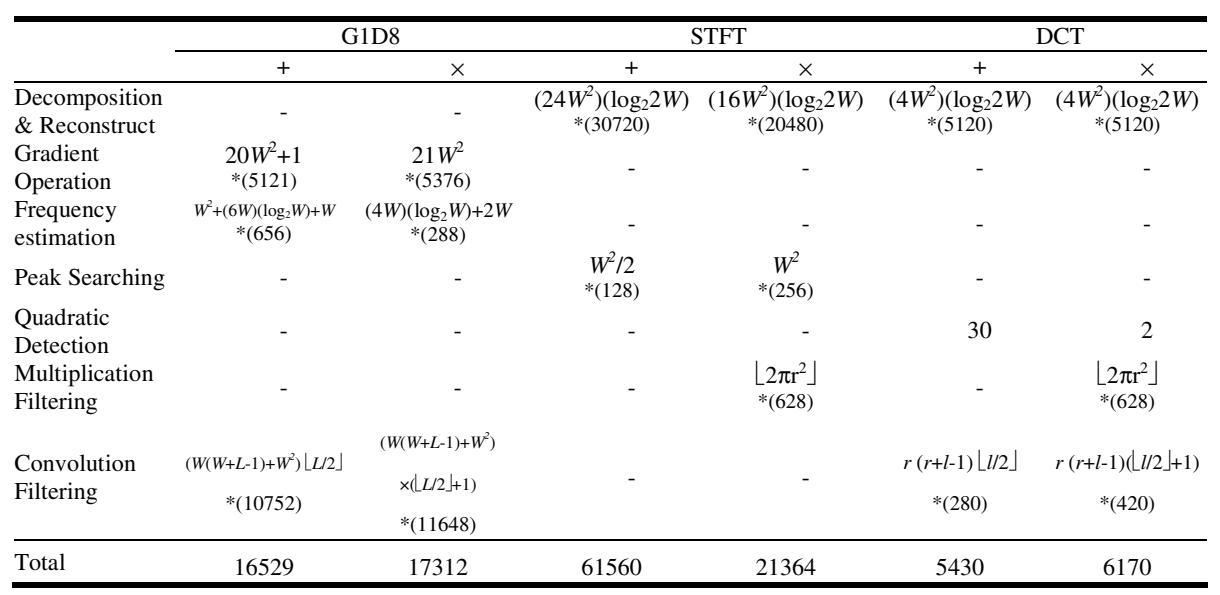




\section{Conclusion}

Performance comparison of block-based fingerprint enhancement was reported in this paper. From Table 1, Short Time Fourier enhancement approach seems to tolerate to most problems except spurious minutiae and fake ridge generation. From Table 2, DCT enhancement approach successfully gives minimum equal error rate (EER) in 5 out of 11 tested databases. Note that DCT approach works well for fair fingerprint quality, but it fails to all FVC2004 databases, which contain many low quality fingerprints with elastic distortion. On the other hand, STFT achieves good results in FVC2004 databases, with minimum EER in 2 out of 4. In addition Spatial Gabor filtering works very well with all synthetic fingerprints (all Db4a). Surprisingly, DCT approach consumes the least computational complexity comparing with STFT and spatial Gabor filtering.

Acknowledgments. This work was partially supported by Department of Electrical Engineering, Kasetsart University, Thailand Research Fund (TRF) through the Royal Golden Jubilee Ph.D. Program (Grant No.PHD/0017/2549), and the Commission on Higher Education through the TRF Research Scholar (Grant No. RMU4980027).

\section{References}

1. Maltoni, D., Maio, D., Jain, A.K., Prabhakar, S.: Handbook of Fingerprint Recognition. Springer, Heidelberg (2003)

2. Hong, L., Wang, Y., Jain, A.K.: Fingerprint Image Enhancement: Algorithm and Performance Evaluation. IEEE Trans. on Pattern Analysis and Machine Intelligence 20(8), $777-$ 789 (1998)

3. Areekul, V., Watchareeruetai, U., Tantaratana, S.: Fast Separable Gabor Filter for Fingerprint Enhancement. In: Zhang, D., Jain, A.K. (eds.) ICBA 2004. LNCS, vol. 3072, pp. 403-409. Springer, Heidelberg (2004)

4. Areekul, V., Watchareeruetai, U., Suppasriwasuseth, K., Tantaratana, S.: Separable Gabor filter realization for fast fingerprint enhancement. In: Proc. Int. Conf. on Image Processing (ICIP 2005), pp. III-253-III-256 (2005)

5. Chikkerur, S., Cartwright, A.N., Govindaraju, V.: Fingerprint Enhancement Using STFT Analysis. Pattern Recognition 40, 198-211 (2007)

6. Jirachaweng, S., Areekul, V.: Fingerprint Enhancement Based on Discrete Cosine Transform. In: Lee, S.-W., Li, S.Z. (eds.) ICB 2007. LNCS, vol. 4642, pp. 96-105. Springer, Heidelberg (2007)

7. Areekul, V., Suppasriwasuseth, K., Jirachawang, S.: The New Focal Point Localization for Fingerprint Registration. In: Proc. Int. Conf. on Image Pattern Recognition (ICPR 2006), vol. 4, pp. 497-500 (2006)

8. Leelasawassuk, T., Areekul, V.: Looped Minutiae Matching in Fingerprint Verification. In: Proceedings of International Workshop on Advanced Image Technology (IWAIT 2007), pp. 924-928 (2007)

9. Leelasawassuk, T.: Fingerprint Matching Using a Focal Point as a Reference Point. Master of Engineering Thesis, Department of Electrical Engineering, Kasetsart University, Thailand (2007)

10. http://bias.csr.unibo.it 